\title{
Pituitary MRI characteristics in 297 acromegaly patients based on T2-weighted sequences
}

Iulia Potorac, Patrick Petrossians, Adrian F Daly, Franck Schillo ${ }^{1}$, Claude Ben Slama ${ }^{2}$, Sonia Nagi ${ }^{3}$, Mouna Sahnoun ${ }^{4}$, Thierry Brue ${ }^{4}$, Nadine Girard ${ }^{5}$, Philippe Chanson ${ }^{6}$, Ghaidaa Nasser ${ }^{7}$, Philippe Caron ${ }^{8}$, Fabrice Bonneville ${ }^{9}$, Gérald Raverot ${ }^{10}$, Véronique Lapras' ${ }^{11}$, François Cotton ${ }^{11}$, Brigitte Delemer ${ }^{12}$, Brigitte Higel ${ }^{13}$, Anne Boulin ${ }^{14}$, Stéphan Gaillard ${ }^{15}$, Florina Luca ${ }^{16}$, Bernard Goichot ${ }^{16}$, Jean-Louis Dietemann" ${ }^{17}$, Albert Beckers and Jean-François Bonneville

Department of Endocrinology, Centre Hospitalier Universitaire de Liège, Université de Liège, Domaine Universitaire du Sart Tilman, 4000 Liège, Belgium

${ }^{1}$ Department of Endocrinology, CHU Besançon, Besançon, France

Departments of ${ }^{2}$ Endocrinology, and ${ }^{3}$ Neuroradiology, School of Medicine, Tunis University, Tunis, Tunisia

Departments of ${ }^{4}$ Endocrinology, and ${ }^{5}$ Neuroradiology, CHU Marseille, Marseille, France

Departments of ${ }^{6}$ Endocrinology, and ${ }^{7}$ Neuroradiology, CHU Bicêtre, Le Kremlin-Bicêtre, France

${ }^{8}$ Departments of Endocrinology, and ${ }^{9}$ Neuroradiology, CHU Toulouse, Toulouse, France

Departments of ${ }^{10}$ Endocrinology, and ${ }^{11}$ Radiology, Hospices Civils de Lyon, Lyon, France

Departments of ${ }^{12}$ Endocrinology, and ${ }^{13}$ Neuroradiology, CHU Reims, Reims, France

Departments of ${ }^{14}$ Neuroradiology, and ${ }^{15}$ Neurosurgery, $\mathrm{CH}$ Foch, Suresnes, France

Departments of ${ }^{16}$ Endocrinology, and ${ }^{17}$ Neuroradiology, CHU Strasbourg, Strasbourg, France

Correspondence

should be addressed

to A Beckers

Email

albert.beckers@chu.ulg.ac.be

\begin{abstract}
Responses of $\mathrm{GH}$-secreting adenomas to multimodal management of acromegaly vary widely between patients. Understanding the behavioral patterns of $\mathrm{GH}$-secreting adenomas by identifying factors predictive of their evolution is a research priority. The aim of this study was to clarify the relationship between the T2-weighted adenoma signal on diagnostic magnetic resonance imaging (MRI) in acromegaly and clinical and biological features at diagnosis. An international, multicenter, retrospective analysis was performed using a large population of 297 acromegalic patients recently diagnosed with available diagnostic MRI evaluations. The study was conducted at ten endocrine tertiary referral centers. Clinical and biochemical characteristics, and MRI signal findings were evaluated. T2-hypointense adenomas represented $52.9 \%$ of the series, were smaller than their T2-hyperintense and isointense counterparts $(P<0.0001)$, were associated with higher IGF1 levels $(P=0.0001)$, invaded the cavernous sinus less frequently $(P=0.0002)$, and rarely caused optic chiasm compression $(P<0.0001)$. Acromegalic men tended to be younger at diagnosis than women $(P=0.067)$ and presented higher IGF1 values $(P=0.01)$. Although in total, adenomas had a predominantly inferior extension in $45.8 \%$ of cases, in men this was more frequent $(P<0.0001)$, whereas in women optic chiasm compression of macroadenomas occurred more often $(P=0.0067)$. Most adenomas (45.1\%) measured between 11 and $20 \mathrm{~mm}$ in maximal diameter and bigger adenomas were diagnosed at younger ages $(P=0.0001)$. The T2-weighted signal differentiates GH-secreting adenomas into subgroups with
\end{abstract}
Key Words
- pituitary adenoma
- acromegaly
- T2-weighted signal

- MRI 
particular behaviors. This raises the question of whether the T2-weighted signal could represent a factor in the classification of acromegalic patients in future studies.

\section{Introduction}

Acromegaly is caused by excess secretion of growth hormone $(\mathrm{GH})$, secreted in most cases by a pituitary adenoma (Melmed 2009). GH-secreting adenomas are the second most frequent type of functional pituitary adenomas (Daly et al. 2006, Fernandez et al. 2010) and usually present as macroadenomas on magnetic resonance imaging (MRI) at diagnosis (Petrossians et al. 2012). Unlike other types of pituitary adenomas, which are usually hyperintense on T2-weighted sequences (e.g. prolactinoma) (Hagiwara et al. 2003, Bonneville et al. 2005), the T2-weighted signal intensity of GH-secreting adenomas varies. This potentially important aspect of the MRI characteristics of GH-secreting pituitary adenomas is particularly unclear, as many historical studies suffered from low-quality images or the absence of T2-weighted sequences.

Evaluation of the behavior and responses to treatment of GH-secreting pituitary adenomas is based on clinical, biochemical, imaging, genetic, and pathological characteristics, with the latter two being the most informative currently. However, germline DNA anomalies, such as mutations in AIP or MEN1 genes, are infrequent in the setting of acromegaly (Daly et al. 2009, Cuny et al. 2013). Pathological analysis contributes important information, but is only available after pituitary surgery. Therefore, in the vast majority of sporadic cases of acromegaly neither genetic nor pathological data can be employed at diagnosis to predict adenoma aggressiveness. The image resolution of pituitary MRI has been improved in recent years and it is currently a fundamental part of the diagnostic work-up of any acromegaly patient. As a noninvasive technique performed at diagnosis, high-resolution pituitary MRI could represent a very good candidate for helping to better characterize GH-secreting pituitary adenomas and predict subsequent pathological and clinical behavior. Results of previous research have indicated a relationship between the intensity of T2-weighted signal, adenoma characteristics (Hagiwara et al. 2003), pathology (Hagiwara et al. 2003, Heck et al. 2012, Bakhtiar et al. 2014), and response to somatostatin analog treatment before (Heck et al. 2012) or after surgery (Puig-Domingo et al. 2010). However, these studies were performed on small series and results have been inconsistent.
In this context, we have performed the current retrospective analysis of a large population of acromegalic patients, aiming to clarify the relationship between the T2-weighted adenoma signal and clinical and hormonal characteristics at diagnosis.

\section{Patients and methods}

This was a retrospective multicenter study that included all acromegalic patients diagnosed at ten tertiary referral centers, whose initial MRIs were available for evaluation. The patients were included irrespective of age, sex, or presentation. All MRIs were performed before any medical treatment. Only patients that had a pre-existing confirmed diagnosis of acromegaly were included. In all cases, the diagnosis of acromegaly was based on the biochemical demonstration of a nonsuppressible GH on an oral glucose tolerance test (OGTT) with an insulin-like growth factor 1 (IGF1) level above the age/sex-matched normal for the individual laboratory, and the presence of a pituitary adenoma on MRI. In addition, $75.5 \%$ of the cases were operated upon and a somatotropinoma was confirmed on pathology (the remaining cases were not operated on and received medical and/or radiotherapy).

The study was approved and performed at the centers using Ethics Committee approval of the Centre Hospitalier Universitaire de Liège covering anonymous data collection regarding the study participants.

\section{Patient characteristics}

The following data regarding each patient were recorded: date of birth, sex, date of diagnosis, and estimated evolution of the disease before diagnosis. Each data point was given a study code in order to maintain anonymity.

\section{Imaging criteria}

The diagnostic MRI studies were performed with highresolution techniques ( 1.5 or $3.0 \mathrm{~T}$ ) and in order to be valid for inclusion there had to be at least a T2-weighted coronal sequence, either a T1- or a T2-weighted sagittal sequence, and a gadolinium-enhanced T1-coronal sequence. The

Published by Bioscientifica Ltd 
MRI images were analyzed at each center by a senior neuroradiologist with over 10 years of experience in sellar MRI interpretation. The following characteristics concerning the adenoma were recorded: maximum diameter, lateral invasion of the cavernous sinus, vertical extension (suprasellar and inferior), and T2-weighted signal. The T2-weighted signal of the adenoma was classified as hypointense, isointense, or hyperintense by visual comparison with the normal pituitary tissue (Fig. 1). When normal pituitary tissue was not visible (e.g. in cases with large adenomas), the adenoma signal was compared with that of cerebral grey matter of the temporal lobe. We chose this order based on a pre-existing assessment of T2-signal intensity by means of a region of interest (ROI) analysis of normal pituitary tissue, grey matter, and adenoma tissue. This was performed on a series of 29 patients at the coordinating center, in whom all three of these structures were visible. ROI evaluation of the adenomas compared with the two comparator structures was concordant with the visual evaluation of the signal in 27 out of 29 cases (93\%). In the two cases (7\%) in which the adenomas were visually isointense, the adenoma signal differed little by ROI evaluation, being only slightly inferior to that of normal pituitary tissue and slightly superior to that of grey matter.

Tumors were classified into four groups according to their maximum diameter: i) $\leq 5 \mathrm{~mm}$, ii) $6-10 \mathrm{~mm}$,

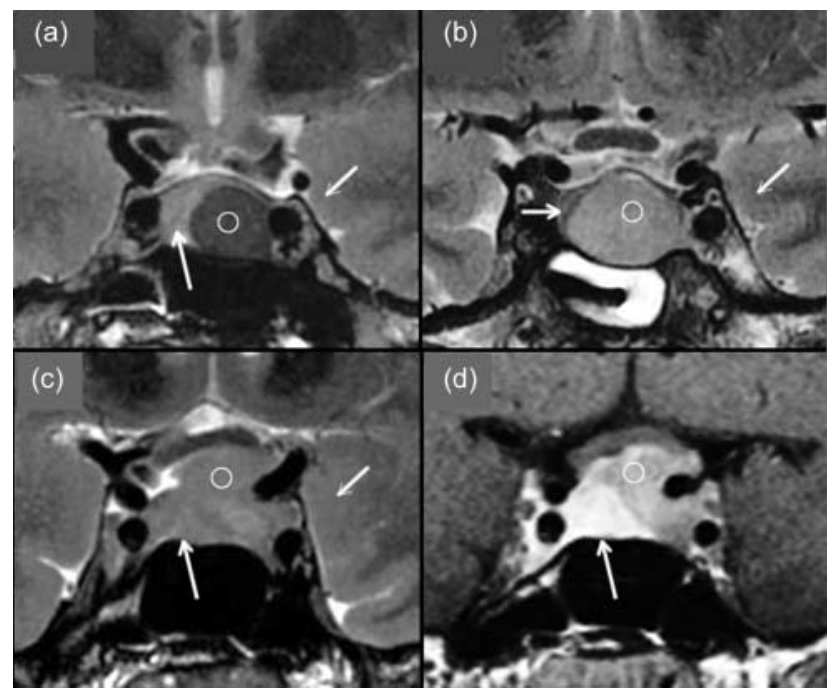

Figure 1

Classification of T2-weighted signal of GH secreting pituitary adenomas in the study: (a) T2-hypointense adenoma, (b) T2-hyperintense adenoma, (c) T2-isointense adenoma, as compared with normal pituitary tissue (thick arrowheads) and temporal lobe grey matter (thin arrowheads). Panel d shows a gadolinium-enhanced T1-weighted of the adenoma illustrated in panel c, differentiating the adenoma (nonenhancing) from the normal pituitary tissue (enhancing). Adenomas are indicated by open white circles. iii) 11-20 $\mathrm{mm}$, and iv) $>20 \mathrm{~mm}$. Lateral invasion of the cavernous sinus was assessed as being present, absent, or uncertain. Other imaging features were noted (presence or absence): intrasellar herniation of the suprasellar subarachnoid spaces, aneurysms of the circle of Willis, and the exact location of such aneurysms when magnetic resonance angiography (MRA) was performed.

The vertical extension was evaluated using two criteria:

i) A visual comparison between the suprasellar and inferior extensions on a sagittal section was encoded as 1 if inferior $>$ suprasellar and as 0 if inferior $=$ suprasellar, or superior $>$ suprasellar.

ii) Presence of optic chiasm compression on a coronal section.

The pneumatization of the sphenoid sinus was classified based on the following:

i) limited to the sinus concha;

ii) if the pneumatization stopped at a vertical line tangent to the sellar tubercle (presellar pneumatization);

iii) if the pneumatization stopped at a vertical line tangential to the root of the dorsum sellae (sellar pneumatization);

iv) if the pneumatization continued further posteriorly of the aforementioned vertical line (retrosellar pneumatization).

\section{Hormonal assays}

The absolute value of IGF1 at diagnosis and the superior limit of the laboratory's normal range adjusted for age and sex were recorded. In order to eliminate variation caused by different assay kits and the different age and sexadjusted normal ranges, the IGF1 levels were expressed as a percentage of the upper limit of the normal range (ULN).

\section{Statistical evaluation}

All the information was encoded as anonymous data by a designated member of each participating center. Univariate data are represented as box-plots, with the limits of the box showing the 25 th and 75 th percentiles, the central line representing the median, and the whiskers 1.5 times the interquartile range of the data. For count variables, statistical comparisons used the $\chi^{2}$ test and for continuous variables, the Mann-Whitney and Kruskal-Wallis tests were used. Data were analyzed using the $\mathrm{R}$ software

Published by Bioscientifica Ltd 
Table 1 Characteristics of GH-secreting adenomas distributed by maximal tumor diameter

\begin{tabular}{|c|c|c|c|c|}
\hline Tumor diameter (mm) & $<\mathbf{5}$ & $6-10$ & $11-20$ & $>20$ \\
\hline Number & 19 & 68 & 134 & 76 \\
\hline Male vs female & 8 vs 11 & 26 vs 42 & 75 vs 59 & 36 vs 40 \\
\hline Age at diagnosis (years) & 55.7 & 52.0 & 47.7 & 41.0 \\
\hline IGF1 (\% ULN) & 204.5 & 250.0 & 305.0 & 265.9 \\
\hline
\end{tabular}

ULN, upper limit of the normal range.

package (R Development Core Team 2011) and graphics were generated using the Lattice package (Sarkar 2008).

\section{Results}

\section{Patient characteristics}

In total, 297 patients were included from the ten participating centers. The patients were equally distributed by gender: 152 females (51.2\%). The median age at diagnosis of acromegaly was 47.5 years. Males had a tendency to be younger at diagnosis than females (46.1 versus 49.0 years respectively; $P=0.067)$. The median disease duration before diagnosis was 5 years (Q1: 3.0, Q3: 10.0) and was similar in males and in females. The median IGF1 percentage of the ULN was $278.4 \%$. In males, median IGF1 was higher than that in females $(300.0 \%$ (Q1: 210.0\%, Q3: 409.5\%) versus 250.0\% (Q1: 180.6\%, Q3: $348.5 \%$ ) respectively; $P=0.01$ ). No other statistically significant or relevant relationships existed among these parameters at baseline.

\section{Tumor characteristics}

Maximum diameter Macroadenomas predominated (70.7\%) among the study population. As given in Table 1, the main subgroup by size consisted of patients with a maximal tumor diameter of 11-20 mm (45.1\%; 134/297).
Patients with larger adenomas were significantly younger at diagnosis $(P=0.0001)$ (Table 1$)$. The levels of IGF1 were also higher in larger adenomas $(P=0.00035)$, although this relationship plateaued above a diameter of $20 \mathrm{~mm}$ (Table 1).

Vertical extension Optic chiasmal compression was found in 53 cases (17.8\% overall) and tended to be more frequent in females as compared with males $(P=0.05)$. This relationship was, however, significant when only macroadenomas were included (Table 2) $(P=0.0067)$. Patients with optic chiasm compression were diagnosed at a significantly younger age $(P=0.00009)$. Adenomas that led to optic chiasm compression had significantly lower levels of IGF1 $(P=0.0066)$.

The direction of extension in the vertical plane was predominantly inferior in 136 cases (45.8\%); this predominantly inferior extension occurred more frequently in males than in females $(P=0.000008)$ (Table 2$)$. Those patients with predominantly inferior adenoma extension were significantly older at diagnosis than those without (49.8 versus 46.0 years respectively; $P=0.029$ ). Among adenomas with predominantly inferior extension, isolated inferior extension (with no suprasellar extension associated) was found in 69 cases (23.2\% of total).

Cavernous sinus invasion Definite invasion of the cavernous sinus was present in 86 cases (28.9\%) and possible invasion was noted in another 14 cases $(4.7 \%)$. Patients with tumors that invaded the cavernous sinus were diagnosed at a younger age $(P=0.0014)$. Invasion of the cavernous sinus was more frequent in larger adenomas, with adenomas of maximum diameter $>20 \mathrm{~mm}$ invading the cavernous sinus in $69.0 \%$ of cases. Also, adenomas with optic chiasm compression significantly more frequently had cavernous sinus invasion $(P=0.000000004)$.

Table 2 Distribution of adenomas by vertical extension relationship with patient and adenoma characteristics

\begin{tabular}{|c|c|c|c|c|c|c|}
\hline & \multicolumn{3}{|c|}{ Optic chiasm compression ${ }^{a}$} & \multicolumn{3}{|c|}{ Predominantly inferior extension } \\
\hline & $\mathrm{Y}$ & $\mathrm{N}$ & $P$ value & $\mathrm{Y}$ & $\mathrm{N}$ & $P$ value \\
\hline Number & 53 & 244 & & 136 & 161 & \\
\hline Male vs female & 19 vs 34 & 126 vs 118 & 0.05 & 86 vs 50 & 59 vs 102 & 0.000008 \\
\hline \multicolumn{7}{|l|}{ Macroadenomas ${ }^{b}$} \\
\hline Male vs female & 19 vs 34 & 92 vs 65 & 0.0067 & 69 vs 38 & 42 vs 61 & 0.0009 \\
\hline Age at diagnosis & 38.0 & 49.0 & 0.00009 & 49.8 & 46.0 & 0.029 \\
\hline IGF1 (\% ULN) & 226.2 & 293.3 & 0.0066 & 290.0 & 263.4 & 0.095 \\
\hline
\end{tabular}

${ }^{a}$ Four adenomas - two microadenomas and two macroadenomas were not included in the analysis due to lack of information regarding optic chiasm compression.

${ }^{b}$ Macroadenomas of $>10 \mathrm{~mm}$ on maximal diameter.

http://erc.endocrinology-journals.org DOI: 10.1530/ERC-14-0305 (c) 2015 Society for Endocrinology Printed in Great Britain
Published by Bioscientifica Ltd 
No other significant relationships were found among patient and tumor characteristics.

\section{MRI characteristics}

T2-weighted signal In T2-weighted sequences, the adenomas were hypointense in 157 cases (52.9\%), hyperintense in 77 cases $(25.9 \%)$, and isointense in 63 cases $(21.2 \%)$. The T2-weighted signal was compared with normal pituitary tissue in 223 cases (75\%) and with the grey matter in the temporal lobe in 74 cases $(25 \%)$. No relationships were found between T2-weighted signal and sex, age at diagnosis, or estimated duration of disease before diagnosis.

T2-hypointense adenomas represented the majority of adenomas in each group up to a diameter $20 \mathrm{~mm}$ (Table 3). However, they accounted for only $27.6 \%$ of the adenomas $>20 \mathrm{~mm}$, whereas $46.0 \%$ of these larger adenomas were hyperintense on T2 (Fig. 2). Patients with T2-hypointense adenomas had significantly higher median IGF1 levels compared with those with T2-isointense and T2-hyperintense adenomas $(P=0.0001)$. Considering the group of T2-hypointense adenomas, a statistically significant relationship was found between tumor diameter and their IGF1 levels $(P=0.00006)$. For T2-hypointense adenomas up to $20 \mathrm{~mm}$, IGF1 levels increased with the maximum diameter of the adenoma.

There was a statistically significant relationship between optic chiasm compression and T2-weighted signal $(P=0.0000001)$. T2-hypointensity was associated with a lower rate of optic chiasm compression than that of either T2-hyperintense or T2-isointense adenomas. This relationship was present in all adenomas $\geq 11 \mathrm{~mm}$ (optic chiasm compression: $9.3 \%$ of T2-hypointense, $45.7 \%$ of T2-hyperintense, $36.3 \%$ of T2-isointense) and also in larger adenomas $>20 \mathrm{~mm}$ (optic chiasm compression: $27.2 \%$ T2-hypointense, $71.4 \%$ T2-hyperintense, $60.8 \%$ T2-isointense).

Cavernous sinus invasion was rare $(n=5)$ in tumors smaller than $11 \mathrm{~mm}$ in maximum diameter; of these, three were T2-hypointense and two were T2-isointense. Among tumors $\geq 11 \mathrm{~mm}$ in maximum diameter, T2-hypointense adenomas invaded the cavernous sinus less frequently (28\%) than T2-hyperintense $(55.9 \%)$ or T2-isointense $(52.2 \%)$ adenomas $(P=0.0002)$ (Table 3$)$.

Other tumor characteristics Most adenomas had extensive pneumatization of the sphenoid sinus: sellar and retrosellar pneumatization were found in 90 and 175 adenomas, respectively ( $79.1 \%$ of all cases), while sinus concha was found only in six cases and presellar pneumatization in 26 patients. Adenomas with predominantly inferior extension had significantly more extended pneumatization $(P=0.0097)$. Intrasellar herniation of the suprasellar subarachnoid spaces was present in 45 cases and absent in 252 cases. The intrasellar presence of the subarachnoid spaces was associated with older age at diagnosis $(P=0.00001)$. In the 46 patients evaluated by MRA, aneurysms of the circle of Willis were infrequent $(n=4)$.

\section{Discussion}

This multicenter study was carried out to address the relationship between diagnostic pituitary MRI features and disease characteristics in 297 acromegaly patients, as this topic has received relatively little attention to date (Hagiwara et al. 2003, Heck et al. 2012, Bakhtiar et al. 2014). We detected a high prevalence of T2-hypointense GH-secreting adenomas, representing over $50 \%$ of all

Table 3 Distribution of adenomas according to T2-weighted signal

\begin{tabular}{|c|c|c|}
\hline & T2-hypointense & T2-isointense \\
\hline Number & 157 & 63 \\
\hline Male vs female & 77 vs 80 & 36 vs 27 \\
\hline Age at diagnosis (years) & 48.7 & 46.1 \\
\hline IGF1 (\% ULN) & 314.7 & 260.2 \\
\hline \multicolumn{3}{|l|}{ Maximum diameter (mm) } \\
\hline$<5$ & 10 & 6 \\
\hline $6-10$ & 40 & 13 \\
\hline $11-20$ & 86 & 24 \\
\hline$>20$ & 21 & 20 \\
\hline Optic chiasm compression & 10 & 16 \\
\hline Cavernous sinus invasion ${ }^{a}$ & 30 & 23 \\
\hline
\end{tabular}

\begin{tabular}{|c|c|}
\hline T2-hyperintense & $P$ value \\
\hline \multicolumn{2}{|l|}{77} \\
\hline 32 vs 45 & NS \\
\hline 45.0 & NS \\
\hline 236.7 & 0.0001 \\
\hline 3 & 0.00001 \\
\hline 15 & \\
\hline 24 & \\
\hline 35 & \\
\hline 27 & 0.0000001 \\
\hline 33 & 0.0002 \\
\hline
\end{tabular}

ULN, upper limit of the normal range.

${ }^{a}$ Analyzed only in adenomas with maximum diameter $>10 \mathrm{~mm}$.

http://erc.endocrinology-journals.org DOI: $10.1530 /$ ERC-14-0305
(C) 2015 Society for Endocrinology Printed in Great Britain
Published by Bioscientifica Ltd. 


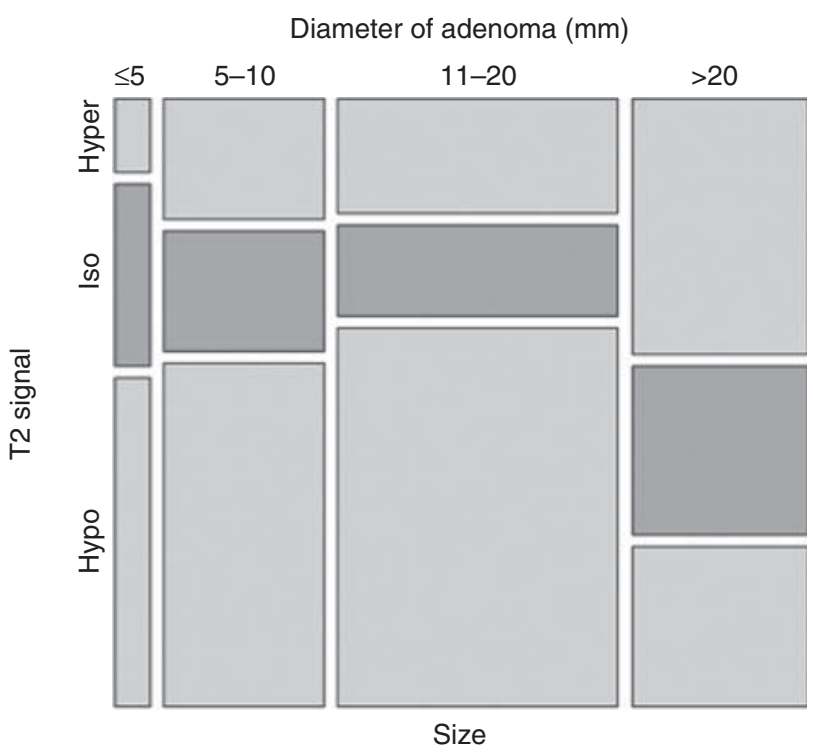

Figure 2

Distribution of the adenomas according to maximal tumor diameter and T2-weighted signal. The width of each column is proportional to the number of cases. The vertical distribution illustrates the proportion of each type of T2-weighted signal within the corresponding adenoma size column. T2-hypointense adenomas are mostly small tumors of up to $20 \mathrm{~mm}$ maximal diameter, as opposed to T2-isointense and T2-hyperintense ones, which form the majority of adenomas with maximal diameter $>20 \mathrm{~mm}$.

cases, which is higher than the 27-40\% reported previously (Hagiwara et al. 2003, Puig-Domingo et al. 2010, Heck et al. 2012, Bakhtiar et al. 2014). This higher proportion may be due to the definition of signal intensity of T2-weighted sequences. The comparator we used to assess the T2weighted signal intensity was normal pituitary tissue; we used the temporal lobe grey matter as the comparator only when no normal pituitary tissue was visualized (e.g. in large tumors). In three other studies (Hagiwara et al. 2003, Puig-Domingo et al. 2010, Heck et al. 2012), T2-hypointensity was defined as a lower intensity than that of white matter, T2-hyperintensity as a higher intensity than that of grey matter, while T2-isointensity was defined as covering the wide range between the intensities of the white and grey matter signals. We believe that definition to be less optimal, as it groups a larger number of tumors as isointense even though their T2-weighted signal is less intense than that of the normal pituitary. In those studies, the lower resolution of T2-weighted sequences in older MRI images may also have made it more difficult to identify the normal pituitary tissue. In one study, the T2-weighted signal of the adenoma was compared with that of white matter. Although the white matter has similar signal intensity to that of the normal pituitary tissue on
T1-weighted sequences, this is not the case in T2-weighted sequences. We believe that future studies should aim to use the T2-weighted signal of normal pituitary tissue, where possible, as the reference comparator for pituitary adenoma signal characteristics.

GH-secreting adenomas are most frequently macroadenomas at diagnosis. Although this was also found in our study, the percentage of microadenomas (30\% in our series) was higher compared with results from previous large studies (Fernandez et al. 2010, Raappana et al. 2010, Petrossians et al. 2012, Gruppetta et al. 2013). This can be explained by the fact that our study included more recently diagnosed patients that have benefited from high-resolution imaging techniques. For instance, very small microadenomas associated with an empty sella - as found in our series - could have been missed on older MRI studies. We found an inverse relationship between tumor size and age at diagnosis, with larger GH-secreting adenomas being diagnosed in younger patients, confirming previous work by our group and others (Lundin et al. 1997, Petrossians et al. 2012). In our study, T2-hypointense adenomas were usually 5-20 mm in diameter at diagnosis, while most macroadenomas $>20 \mathrm{~mm}$ in diameter were $\mathrm{T} 2$-isointense and hyperintense. This is indicative of a higher proliferative potential of T2-isointense and hyperintense adenomas. These larger adenomas appearing at a younger age may indicate more aggressive tumor behavior. One reason for this increased growth might be molecular genetic factors, as this profile of larger adenomas in young patients is shared with AIP-mutation-related adenomas (Beckers et al. 2013). In terms of somatic DNA alterations, GSP oncogeneactivating mutations are the most frequently encountered (Landis et al. 1990). Higher expression of the GS $\alpha$ protein, either in GSP-positive adenomas or in GSP-negative ones, is associated with smaller adenomas (Landis et al. 1990, Spada et al. 1990, Kim et al. 2001, Picard et al. 2007). GSP-positive adenomas seem to have higher $\mathrm{GH}$ secretion and better response to somatostatin analogs (Spada et al. 1990, Barlier et al. 1998). A study to relate genetic pathophysiology to radiological characteristics on MRI would be useful to further explore these possible relationships.

We confirmed in this large series that GH-producing pituitary adenomas have a propensity towards inferior extension (Fig. 3), although not as frequently as other smaller series. As compared with the nearly $46 \%$ of the GH-producing adenomas with predominantly inferior extension in our study, the smaller study by Zada et al. (2010) reported inferior extension in $72 \%$ of 25 $\mathrm{GH}$-secreting pituitary adenomas. Isolated inferior extension, namely adenomas that have no extension above the

Published by Bioscientifica Ltd. 


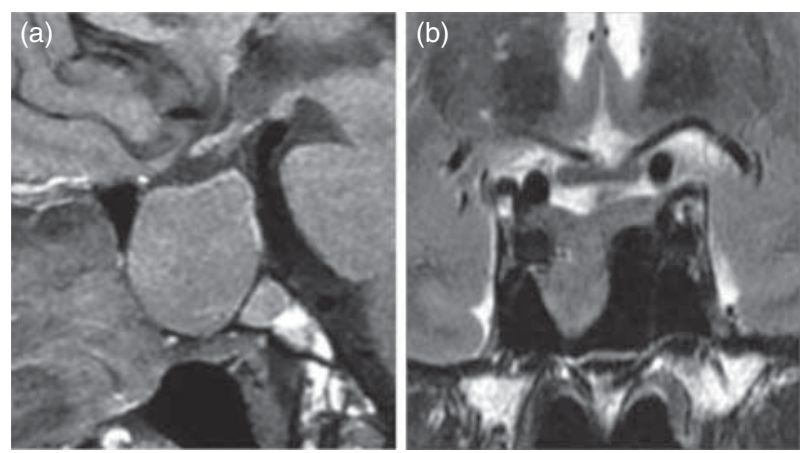

Figure 3

Inferior extension of GH-secreting pituitary adenoma: (a) predominantly inferior extension on sagittal section, (b) isolated inferior extension on coronal section.

sellar diaphragm (Fig. 3), particularly interested us. Zada et al. (2010) determined that $38 \%$ of adenomas with isolated inferior extension were atypical pituitary adenomas under the WHO criteria. However, in our series we found isolated inferior extension in only $23 \%$ of cases. The differences from our results may be due to the small sample size of previous studies and differences in definition of suprasellar extension (Zada et al. 2010). There is also an apparent sex dichotomy concerning vertical extension of GH-secreting adenomas. In male patients, the direction of vertical extension seems to be predominantly inferior.

To our knowledge, this is also the first large MRI study to assess optic chiasm compression in somatotropinomas. We found that GH-secreting pituitary adenomas extend into the opto-chiasmatic cistern relatively rarely, leading to deformation of the optic chiasm in only $17.8 \%$ of cases. These results are in keeping with the results of smaller studies, which indicate that GH-secreting tumors exhibit less suprasellar extension than other pituitary adenomas (Zada et al. 2010). Hagiwara et al. (2003) showed that the mean suprasellar extension was $-0.8 \mathrm{~mm}$ in GH-secreting adenomas as compared with $+4.1 \mathrm{~mm}$ in prolactinomas and $+5.7 \mathrm{~mm}$ in Nonfunctional pituitary adenomas. From a clinical perspective, this decreased tendency to clinically significant suprasellar extension could contribute to the usually long evolution of acromegaly before diagnosis. Females had macroadenomas with optic chiasm compression significantly more frequently than males. We also determined that T2-hypointense adenomas cause optic chiasm compression significantly less frequently than T2-hyperintense and T2-isointense adenomas. We believe that the lesser degree of suprasellar extension is mainly a consequence of these tumors being smaller than T2-isointense/hyperintense adenomas.
The reason for why different pituitary adenomas undergo extension in predominantly different directions is unexplained. A number of possible causes exist for the predominantly inferior extension of GH-secreting pituitary adenomas. A local effect of GH to thicken the collagenous sellar diaphragm could limit superior extension of the pituitary adenoma. However, congenital absence or dehiscence of the sellar diaphragm is so frequent that this explanation seems very unlikely. Passive enlargement of the sella under the direct influence of GH, as is the case for the frontal sinuses and the mastoid cells, is also unlikely because the presence of a GH-secreting microadenoma in a disproportionately large sella is unusual in our experience. We have looked at the intrasellar herniation of the suprasellar subarachnoid spaces as a marker of disproportion between the sellar content and container to confirm this hypothesis. Intrasellar herniation was found in 45/297 (15.2\%) of our patients with a significant correlation with an older age at diagnosis, which indicates that this intrasellar herniation most probably results from spontaneous involution of the adenoma. Initial localization of GHproducing cells in the postero-inferior region of the sella could be a reasonable explanation for the direction of expansion in microadenomas, but this appears unlikely in macroadenomas. Finally, we also found a statistically significant relationship between the predominantly inferior adenoma extension and sphenoid sinus pneumatization $(P=0.0097)$. Hyperpneumatization of the frontal and sphenoid sinuses may occur due to GH-induced bone turnover with apposition of bone along the outer surface of the sinus associated with internal resorption leading to an enlarged sinus cavity (Hagiwara et al. 2003). We propose that this phenomenon could lead to thinning and fragility of the sellar floor, thereby facilitating inferior extension of the GH-secreting pituitary adenomas.

In our series, invasion of the cavernous sinus was diagnosed in nearly $30 \%$ of GH-secreting adenomas, which is higher than that generally reported. This might be accounted for by increased diagnosis of subtle invasion by specialized pituitary neuroradiologists in all centers. Moreover, 3.0 T MRI was available in most participating centers, thereby permitting visualization of the thin medial dural limit and the identification of small intracavernous herniations of adenomatous tissue (usually located posteriorly in the cavernous sinus). This was further facilitated by the use of $2 \mathrm{~mm}$ coronal and axial T2-sequences. We found a significant difference in cavernous sinus invasion according to the different T2-weighted signals. Cavernous sinus invasion was noted

Published by Bioscientifica Ltd. 
in $55.9 \%$ of $\mathrm{T} 2$-hyperintense macroadenomas, and $52.2 \%$ of $\mathrm{T} 2$-isointense macroadenomas, as opposed to only $28 \%$ of T2-hypointense macroadenomas. This is an important observation, as T2-hypointense GH-secreting pituitary adenomas may be more amenable to complete resection as compared with T2-hyperintense and T2isointense adenomas, but this requires further assessment in larger specific studies.

A possible limitation of our study is the absence of a strict quantitative evaluation of the intensity of the T2-weighted signal. The T2-weighted signal of GH-secreting adenomas is a continuous spectrum and the classification into T2-hypointense, isointense, and hyperintense categories is somewhat artificial. This is why quantification would be of utmost interest, but unfortunately is not yet possible. In contrast to the situation in computed tomography, where Hounsfield units are used as an absolute reference system, there is no such quantitative scale for MRI. Another potential limitation is the difficulty of evaluating the degree of heterogeneity in pituitary adenomas corresponding to areas of degenerative changes. Important tumoral heterogeneity could cause difficulties in the assessment of the T2-weighted signal of the adenoma. Another potential limitation is that centralized, blinded reading was not performed in this study. However, all participating neuroradiologists have extensive clinical experience in the interpretation of pituitary MRI and the presence of multiple sources leading to uniform results could also be argued to show consistent interpretation across the study centers. We did not assess prolactin co-secretion at diagnosis as the purpose of our study was to describe a general population of acromegalic patients and not subgroups depending on associated secretion. However, we believe that such a classification could prove interesting in future research, especially in terms of response to treatment.

In conclusion, in this large population of $297 \mathrm{GH}-$ secreting adenomas, we found that T2-weighted sequences could help to classify GH-secreting pituitary adenomas into a T2-hypointense group with smaller adenomas that had less cavernous sinus invasion, less frequent optic chiasm compression and higher IGF1 levels compared with a second group comprising T2-isointense/ hyperintense adenomas. We will further study these groupings by focusing on the correlations with histological subtypes, particularly important being the relationship between granulation pattern and T2-weighted signal, and on whether such imaging characteristics can predict the response to primary somatostatin analog therapy in terms of tumor shrinkage and biological control.

\section{Declaration of interest}

The authors declare that there is no conflict of interest that could be perceived as prejudicing the impartiality of the research reported.

\section{Funding}

The study was supported by the Fonds d'Investissement pour la Recherche Scientifique du Centre Hospitalier de Liège and by an educational grant from Pfizer S.A., Belgium.

\section{References}

Bakhtiar Y, Hanaya R, Tokimura H, Hirano H, Oyoshi T, Fujio S, Bohara M \& Arita K 2014 Geometric survey on magnetic resonance imaging of growth hormone producing pituitary adenoma. Pituitary 17 142-149. (doi:10.1007/s11102-013-0479-z)

Barlier A, Gunz G, Zamora AJ, Morange-Ramos I, Figarella-Branger D, Dufour H, Enjalbert A \& Jaquet P 1998 Prognostic and therapeutic consequences of $\mathrm{G}_{\mathrm{s}} \alpha$ mutations in somatotroph adenomas. Journal of Clinical Endocrinology and Metabolism 83 1604-1610. (doi:10.1210/ jcem.83.5.4797)

Beckers A, Aaltonen LA, Daly AF \& Karhu A 2013 Familial isolated pituitary adenomas (FIPA) and the pituitary adenoma predisposition due to mutations in the aryl hydrocarbon receptor interacting protein (AIP) gene. Endocrine Reviews 34 239-277. (doi:10.1210/er.2012-1013)

Bonneville J-F, Bonneville F \& Cattin F 2005 Magnetic resonance imaging of pituitary adenomas. European Radiology 15 543-548. (doi:10.1007/ s00330-004-2531-x)

Cuny T, Pertuit M, Sahnoun-Fathallah M, Daly A, Occhi G, Odou MF, Tabarin A, Nunes ML, Delemer B, Rohmer V et al. 2013 Genetic analysis in young patients with sporadic pituitary macroadenomas: besides AIP don't forget MEN1 genetic analysis. European Journal of Endocrinology/ European Federation of Endocrine Societies 168 533-541. (doi:10.1530/ EJE-12-0763)

Daly AF, Rixhon M, Adam C, Dempegioti A, Tichomirowa MA \& Beckers A 2006 High prevalence of pituitary adenomas: a cross-sectional study in the province of Liege, Belgium. Journal of Clinical Endocrinology and Metabolism 91 4769-4775. (doi:10.1210/jc.2006-1668)

Daly AF, Tichomirowa MA \& Beckers A 2009 Genetic, molecular and clinical features of familial isolated pituitary adenomas. Hormone Research 71 (Suppl 2) 116-122. (doi:10.1159/000192448)

Fernandez A, Karavitaki N \& Wass JA 2010 Prevalence of pituitary adenomas: a community-based, cross-sectional study in Banbury (Oxfordshire, UK). Clinical Endocrinology 72 377-382. (doi:10.1111/ j.1365-2265.2009.03667.x)

Gruppetta M, Mercieca C \& Vassallo J 2013 Prevalence and incidence of pituitary adenomas: a population based study in Malta. Pituitary 16 545-553. (doi:10.1007/s11102-012-0454-0)

Hagiwara A, Inoue Y, Wakasa K, Haba T, Tashiro T \& Miyamoto T 2003 Comparison of growth hormone-producing and non-growth hormoneproducing pituitary adenomas: imaging characteristics and pathologic correlation. Radiology 228 533-538. (doi:10.1148/radiol.2282020695)

Heck A, Ringstad G, Fougner SL, Casar-Borota O, Nome T, RammPettersen J \& Bollerslev J 2012 Intensity of pituitary adenoma on T2-weighted magnetic resonance imaging predicts the response to octreotide treatment in newly diagnosed acromegaly. Clinical Endocrinology 77 72-78. (doi:10.1111/j.1365-2265.2011.04286.x)

Kim HJ, Kim MS, Park YJ, Kim SW, Park DJ, Park KS, Kim SY, Cho BY, Lee HK, Jung HW et al. 2001 Prevalence of Gs alpha mutations in Korean patients with pituitary adenomas. Journal of Endocrinology 168 221-226. (doi:10.1677/joe.0.1680221)
(C) 2015 Society for Endocrinology Printed in Great Britain 
Landis CA, Harsh G, Lyons J, Davis RL, McCormick F \& Bourne HR 1990 Clinical characteristics of acromegalic patients whose pituitary tumors contain mutant Gs protein. Journal of Clinical Endocrinology and Metabolism 71 1416-1420. (doi:10.1210/jcem-71-6-1416)

Lundin P, Engström BE, Karlsson F \& Burman P 1997 Long-term octreotide therapy in growth hormone-secreting pituitary adenomas: evaluation with serial MR. American Journal of Neuroradiology 18 765-772.

Melmed S 2009 Acromegaly pathogenesis and treatment. Journal of Clinical Investigation 119 3189-3202. (doi:10.1172/JCI39375)

Petrossians P, Tichomirowa MA, Stevenaert A, Martin D, Daly AF \& Beckers A 2012 The Liege Acromegaly Survey (LAS): a new software tool for the study of acromegaly. Annales d'Endocrinologie 73 190-201. (doi:10.1016/j.ando.2012.05.001)

Picard C, Silvy M, Gerard C, Buffat C, Lavaque E, Figarella-Branger D, Dufour H, Gabert J, Beckers A, Brue T et al. 2007 Gs alpha overexpression and loss of $\mathrm{G}_{\mathrm{s}} \alpha$ imprinting in human somatotroph adenomas: association with tumor size and response to pharmacologic treatment. International Journal of Cancer. Journal International du Cancer 121 1245-1252. (doi:10.1002/ijc.22816)

Puig-Domingo M, Resmini E, Gomez-Anson B, Nicolau J, Mora M, Palomera E, Marti C, Halperin I \& Webb SM 2010 Magnetic resonance imaging as a predictor of response to somatostatin analogs in acromegaly after surgical failure. Journal of Clinical Endocrinology and Metabolism 95 4973-4978. (doi:10.1210/jc.2010-0573)

Raappana A, Koivukangas J, Ebeling T \& Pirila T 2010 Incidence of pituitary adenomas in Northern Finland in 1992-2007. Journal of Clinical Endocrinology and Metabolism 95 4268-4275. (doi:10.1210/jc.20100537)

R Development Core Team 2011 R: A Language and Environment for Statistical Computing. Vienna, Austria: R Foundation for Statistical Computing.

Sarkar D 2008 Lattice: Multivariate Data Visualization with R. New York: Springer.

Spada A, Arosio M, Bochicchio D, Bazzoni N, Vallar L, Bassetti M \& Faglia G 1990 Clinical, biochemical, and morphological correlates in patients bearing growth hormone-secreting pituitary tumors with or without constitutively active adenylyl cyclase. Journal of Clinical Endocrinology and Metabolism 71 1421-1426. (doi:10.1210/jcem-716-1421)

Zada G, Lin N \& Laws ER Jr 2010 Patterns of extrasellar extension in growth hormone-secreting and nonfunctional pituitary macroadenomas. Neurosurgical Focus 29 E4. (doi:10.3171/2010.7.FOCUS10155)

Received in final form 23 December 2014

Accepted 1 January 2015

Made available online as an Accepted Preprint

2 January 2015
(C) 2015 Society for Endocrinology Printed in Great Britain
Published by Bioscientifica Ltd. 